\title{
A preliminary score for the assessment of disease activity in hereditary recurrent fevers: results from the AIDAI (Auto-Inflammatory Diseases Activity Index) Consensus Conference
}

\author{
Maryam Piram, ${ }^{1}$ Joost Frenkel, ${ }^{2}$ Marco Gattorno, ${ }^{3}$ Seza Ozen, ${ }^{4}$ Helen J Lachmann, ${ }^{5}$ \\ Raphaela Goldbach-Mansky, ${ }^{6}$ Véronique Hentgen, ${ }^{7}$ Bénédicte Neven, ${ }^{8}$ Katia Stankovic \\ Stojanovic ${ }^{9}$ Anna Simon, ${ }^{10}$ Jasmin Kuemmerle-Deschner, ${ }^{11}$ Hal Hoffman, ${ }^{12}$ Silvia \\ Stojanov, ${ }^{13}$ Agnès Duquesne, ${ }^{14}$ Pascal Pillet, ${ }^{15}$ Alberto Martini, ${ }^{3}$ Jacques Pouchot, ${ }^{16}$ \\ Isabelle Koné-Paut ${ }^{1}$; on behalf of the EUROFEVER and EUROTRAPS networks
}

For numbered affiliations see end of article

\section{Correspondence to}

Professor Isabelle Koné-Paut, Department of Pediatrics and Pediatric Rheumatology, National Reference Center for Auto-Inflammatory Disorders, Bicêtre University Hospital, Paris XI University, 78 rue du Général Leclerc, 94270 Le Kremlin Bicêtre, France;

isabelle.kone-paut@bct.aphp.fr

Accepted 19 September 2010 Published Online First 15 November 2010

\begin{abstract}
Background The systemic autoinflammatory disorders (SAID) share many clinical manifestations, albeit with variable patterns, intensity and frequency. A common definition of disease activity would be rational and useful in the management of these lifelong diseases. Moreover, standardised disease activity scores are required for the assessment of new therapies in constant development. The aim of this study was to develop preliminary activity scores for familial Mediterranean fever, mevalonate kinase deficiency, tumour necrosis factor receptor-1associated periodic syndrome and cryopyrin-associated periodic syndromes (CAPS).
\end{abstract}

Methods The study was conducted using two wellrecognised consensus formation methods: the Delphi technique and the nominal group technique. The results from a two-step survey and data from parent/patient interviews were used as preliminary data to develop the agenda for a consensus conference to build a provisional scoring system.

Results 24 of 65 experts in SAID from 20 countries answered the web questionnaire and 16 attended the consensus conference. There was consensus agreement to develop separate activity scores for each disease but with a common format based on patient diaries. Fever and disease-specific clinical variables were scored according to their severity. A final score was generated by summing the score of all the variables divided by the number of days over which the diary was completed. Scores varied from 0 to 16 (0-13 in CAPS). These scores were developed for the purpose of clinical studies but could be used in clinical practice.

Conclusion Using widely recognised consensus formation techniques, preliminary scores were obtained to measure disease activity in four main SAID. Further prospective validation study of this instrument will follow.

\section{INTRODUCTION}

The current definition of systemic autoinflammatory diseases (SAID) covers a wide spectrum of disorders, all manifesting with excessive systemic and organ-specific inflammation ranging from rare monogenic hereditary recurrent fevers (HRFs) to a growing number of more common multifactorial diseases such as Crohn's and Behçet's diseases. ${ }^{1}$ Traditionally, the HRFs include familial Mediterranean fever (FMF), mevalonate kinase deficiency (MKD), tumour necrosis factor receptor-associated periodic syndrome (TRAPS) and cryopyrin-associated periodic syndromes (CAPS). More recently, three other monogenic entities with variable development of fever have been added to this group: PAPA (pustulosis, acne, pyoderma gangrenosum, arthritis) syndrome, NLRP12-associated periodic syndrome and DIRA (interleukin-1 receptor antagonist deficiency) syndrome. Febrile attacks of HRF are associated with acute biochemical inflammation. Systemic amyloid A (AA) amyloidosis represents a serious longterm complication. Blood tests show leucocytosis and elevated acute phase reactants (eg, C reactive protein (CRP) and serum amyloid $A$ protein (SAA)). However, specific diagnosis relies on identification of mutations in the relevant genes (listed online at http://fmf.igh.cnrs.fr/ISSAID/infevers) and/or the recognition of specific clinical disease manifestations. All known proteins involved in hereditary SAID induce abnormalities of innate immunity, many of which through participating more or less directly in interleukin-1 $\beta$ (IL-1 $\beta$ ) regulation. Mutations in these proteins are thought to result in increased or prolonged secretion of this proinflammatory cytokine. Although IL-1 $\beta$ is not the only effector cytokine in driving the inflammatory process in all of these disorders, therapeutic approaches targeting the IL-1 $\beta$ pathway have shown dramatic responses in almost all HRFs and especially in CAPS where double-blind placebo controlled studies have proven efficacy. ${ }^{2} 3$

While there are now increasing possibilities for targeted therapies in SAID, there is no consensus agreement on any outcome measures in this field. Standardised disease activity scores are required to assess new medications ${ }^{4}$ by using variables which can change over time. As SAID have similar clinical manifestations, albeit with variable patterns, intensity and frequency, ${ }^{5}$ we hypothesised that a common definition of disease activity scores would be a rational approach to improve the management of these lifelong diseases. This study aims to identify and score candidate measures of disease activity for the four main HRFs (FMF, MKD, CAPS and TRAPS). 


\section{METHODS}

We used two widely accepted consensus formation methods ${ }^{6-10}$ specifically designed to combine opinions from a group of experts in a particular field: the Delphi technique and the nominal group technique (NGT). The Delphi technique uses a series of well-defined questionnaire-based surveys. NGT is a structured face-to-face meeting designed to facilitate the consensus on the topic field of study. This technique encourages equal participation from group members and results in a set of prioritised solutions or recommendations. A two-step Delphi technique was used to reduce potential variables to a manageable number and these were then further defined by a NGT at a consensus meeting to develop preliminary scores.

The project was conducted in four steps.

\section{Step 1: Delphi preparation meeting (Dec 2008)}

The steering committee was composed by six international experts in SAID (4 French, 1 Italian, 1 Dutch) from French reference centres for SAID and EUROFEVER network partners, a methodologist and a study coordinator. During a first face-toface meeting they generated and ranked preliminary variables reflecting disease activity for the four main HRF: FMF, MKD, CAPS and TRAPS. At the end of this process, 22 variables ranked $>2$ and belonging to four different domains (clinical, biological, global assessment and quality of life) were chosen and submitted to 65 preselected international experts.

\section{Step 2: Delphi survey (Feb/Aug 2009)}

Similarly to Ruperto et al, ${ }^{7}$ we used a two-phase anonymous online survey (http://www.surveymonkey.com/) to select the variables which can be used to assess whether a patient has an active SAID. The surveys involved 65 experienced practising experts for SAID, members of the PReS and ISSAID societies from 20 different countries. In the first survey, physicians were asked to choose relevant variables from the 22 selected by the study group. The number of selected variables could vary from 0 to 22 and new variables could be suggested. Experts also answered 13 targeted questions regarding evaluation of SAID activity and chose which aspects of some of the previous variables were relevant (eg, frequency, intensity, duration). The second survey consisted of variables and items for which no consensus was obtained ( $<80 \%$ agreement) in the first round and new variables and items suggested by experts. However, experts refined these variables for each of the SAID separately. CAPS was subdivided into familial cold autoinflammatory syndrome (FCAS), Muckle-Wells syndrome (MWS) and chronic infantile neurological, cutaneous, articular (CINCA) also known as neonatal multisystem inflammatory disease (NOMID). To increase the response to the Delphi survey, several recall emails were sent to the experts. The Delphi survey was anonymous and only those who replied to the first round could participate in the second round.

\section{Step 3: Parent and patient interviews (Sept/0ct 2009)}

A standardised questionnaire was completed by parents/patients with one of the four SAID seen in four investigator centres in three countries over a 2 -month period. In the event of language barriers, the investigator physician translated the questionnaire. Initially, patients/parents were asked to provide the three most relevant signs or symptoms indicating active disease. They then rated 35 potential SAID-related clinical signs or symptoms according to importance for perceived disease activity (no/mild/ moderate/important/very important). Patients/parents also had the opportunity to suggest and rate other variables that seemed relevant to them. Finally, they gave their opinion about the impact of the disease on their daily life. For the analysis, all variables were scored from 0 to 4 points according to their importance. The three variables identified as most relevant by the patient were given an additional 2-point weighting. An average score for each variable was then calculated.

\section{Step 4: Consensus conference (Nov 2009)}

A 3-day consensus conference was held in Paris, France. Participants (listed as co-authors) were 16 international adult and paediatric physicians from seven countries whose expertise covered the four SAID, two methodologists and a secretary. The main goal of the conference was to define, through NGT, the domains and variables to include in the provisional activity score. For each question, participants spent several minutes individually considering all the possible ideas. The ideas were collected by sharing them in a 'round robin' format (one response per person each time). Consensus was obtained if 13 of the 16 experts provided the same response. Otherwise, participants evaluated suggested ideas, shared thoughts, deleted duplicate items and individually voted for the best response until reaching a consensus. The results of the Delphi surveys and interviews with parents/patients were considered, but the expert final opinion was used as the gold standard. To establish disease-specific activity scores, a brief review of the specific manifestations of each SAID was presented and then experts were divided into disease-specific groups. Each group worked separately and then presented its conclusions to all the participants. All experts discussed the proposals and the final activity score was developed by a second consensus.

\section{RESULTS \\ Delphi surveys}

The response rate to the first round was 37\% (24/65 experts answered the questionnaire). The experts agreed on the necessity of global disease assessment scores. Among the 22 variables initially suggested, 8 reached consensus and the experts agreed that frequency, duration of joint symptoms and the number of affected joints better assess joint disease activity (box 1). The experts suggested five new variables after the first round (erythrocyte sedimentation rate (ESR), S100, hearing loss, urological symptoms and oral lesions), but above all they suggested some items to better assess joint disease activity, skin activity and CNS activity (data not shown). Nineteen of 24 experts completed the second survey which included these new variables and items.

Box 1 Variables selected by consensus $(\geq 80 \%$ ) for all systemic autoinflammatory diseases during first round of the Delphi survey

- Fever $92 \%$

- Mean duration of attacks $92 \%$

- Number of attacks/period $92 \%$

- Joint symptoms $92 \%$

- Frequency of attacks $90 \%$

- Duration of attacks $95 \%$

- Number of affected joints $80 \%$

- Skin rash $92 \%$

- Serositis $92 \%$

- C reactive protein $92 \%$

- Systemic amyloid A protein $80 \%$ 
Variables selected by consensus ( $\geq 80 \%$ ) during the second round of the Delphi survey are shown in table 1.

\section{Patient and parent interviews}

Nineteen patients/parents from three countries participated in a standardised interview. One questionnaire was incomplete so 18 questionnaires were analysed (8 women and 10 men aged 2.547.4 years (mean 13.7, median 12.1); 3 with FMF, 7 with MKD, 4 with TRAPS, 2 with MWS and 2 with CINCA/NOMID). For each variable the mean score varied from 0 to 6 . A mean score of $\geq 4$ identified variables which the patients considered to be very important. These were: fever for FMF; fever, lymphadenopathy, abdominal pain and anorexia for MKD; fever, abdominal pain, fatigue, intensity of attacks and intensity of fever for TRAPS; fever and fatigue for MWS; presence of skin rash and its frequency for CINCA/NOMID.

\section{Consensus conference}

The first part of the consensus conference was devoted to the analysis and discussion of the preliminary data from the Delphi surveys and interviews with patients and parents.

There was a consensus to build a scoring system which would be a useful instrument in clinical trials and to develop separate activity scores for each disease with a shared format. The clinical disease activity scores would be obtained from a patient diary completed during attacks. Fever was scored as absent (0) or present (1) and disease-specific clinical variables were scored according to their severity from 0 to 3 .

\section{Reports from small group workshops regarding each SAID FMF}

FMF is caused by mutations in pyrin which cause recurrent attacks characterised by fever and serositis. Serositis may manifest itself as abdominal pain, chest pain, arthritis/arthralgia or myalgia. ${ }^{11-16}$ In our Delphi survey, physicians agreed with $>90 \%$ consensus that fever, joint symptoms, serositis, chest and skin symptoms as well as the number and duration of attacks were important in the evaluation of FMF disease activity. Thus, the study group agreed that these features should be reflected in the final activity score for FMF. Patients with FMF may also have symptoms between disease flares such as arthralgia, myalgia and an erysipelas-like rash, which can also be important measures of disease activity. ${ }^{12} 1415$

\section{MKD}

$\mathrm{MKD}$ is caused by mutations in the mevalonate kinase gene inducing recurrent attacks characterised by fever, gastrointestinal symptoms, lymphadenopathy and musculoskeletal symptoms. There was consensus that the frequency, intensity and duration of attacks should be considered in an activity score. Gastrointestinal symptoms are the most prominent and distressing feature of MKD attacks, ${ }^{17}$ and both experts and patients and their parents gave them priority ranking. Thus, the MKD provisional score includes three gastrointestinal features: abdominal pain, diarrhoea and nausea/vomiting. Skin symptoms were not included in the final score since group members felt that $\mathrm{MKD}$ related skin symptoms were not always related to the activity of the disease.

\section{TRAPS}

TRAPS is caused by mutations in the TNFRSF1A gene and results in recurrent but typically infrequent attacks of fever, varying in duration from a few days to several weeks. ${ }^{18}$ The most characteristic symptoms in association with fever include severe abdominal pain, localised myositis and skin manifestations (erythema, periorbital oedema). As the whole clinical spectrum of

Table 1 Variables selected by consensus ( $\geq 80 \%)$ during second round of the Delphi survey

\begin{tabular}{|c|c|c|c|c|c|c|}
\hline \multirow[b]{2}{*}{ Variables } & \multirow[b]{2}{*}{ FMF (\%) } & \multirow[b]{2}{*}{ TRAPS (\%) } & \multirow[b]{2}{*}{ MKD (\%) } & \multicolumn{3}{|l|}{ CAPS } \\
\hline & & & & FCAS $(\%)$ & MWS (\%) & NOMID (\%) \\
\hline Fatigue & & 82 & 81 & 87 & 87 & 82 \\
\hline Number of days of illness in the past 3 months & 94 & 88 & 100 & 93 & 87 & 82 \\
\hline Intensity of attacks & 89 & & 81 & & & \\
\hline Arthritis & 100 & & & & & \\
\hline Arthralgia & & & 87 & & 80 & 87 \\
\hline Measure of joint damage & & & & & & 94 \\
\hline Presence absence of skin rash & 94 & 81 & 82 & 100 & 100 & 88 \\
\hline Intensity of skin rash & & & & 93 & 87 & 88 \\
\hline Frequency of skin rash & & & & 87 & 80 & \\
\hline Number of days with skin rash in a month & & & & 93 & 93 & 82 \\
\hline Chest symptoms & 94 & & & & & \\
\hline Muscle symptoms & 82 & & & & & \\
\hline Gastrointestinal symptoms & & 100 & & & & \\
\hline Painful lymphnodes & & & 94 & & & \\
\hline Hearing loss & & & & & 93 & 82 \\
\hline Eye symptoms & & & & & & 82 \\
\hline Headaches & & & & & & 82 \\
\hline Brain MRI & & & & & & 82 \\
\hline Cognitive function test & & & & & & 94 \\
\hline Funduscopy & & & & & & 88 \\
\hline Limitation of daily activity & 89 & & 87 & 87 & 80 & 81 \\
\hline Days of school /work loss & 83 & & 81 & & & \\
\hline Global DAS physician & 83 & & 81 & & & 88 \\
\hline Global DAS patient & & & & & & 82 \\
\hline$E S R$ & 83 & & & & & \\
\hline
\end{tabular}

New variables/items suggested by experts after the first round are shown in italics.

CAPS, cryopyrin-associated periodic syndromes; CINCA/NOMID, chronic infantile neurological, cutaneous, articular/neonatal onset multisystem inflammatory disease; DAS, Disease

Activity Score; ESR, erythrocyte sedimentation rate; FCAS, familial cold autoinflammatory syndrome; FMF, familial Mediterranean fever; MKD, mevalonate kinase deficiency; MWS,

Muckle-Wells syndrome; TRAPS, TNF receptor-1-associated periodic syndrome. 
TRAPS is very heterogeneous and not yet completely defined, especially in patients carrying non-cysteine TNFR mutations, the study group decided to add an assessment of overall TRAPSrelated symptoms in the disease activity score. In addition to the requirement for pain-relieving medications, the group decided to quantify the amount of administered corticosteroids, even though these two were not included in the calculation of the final score.

\section{CAPS}

CAPS comprises a spectrum of three well-recognised clinical entities that increase in severity from FCAS to MWS and CINCA/NOMID syndrome. ${ }^{19}$ While symptoms in FCAS are classically triggered by cold exposure and feelings of chills that are most pronounced in the evening, symptoms in CINCA/ NOMID are typically continuous and often headaches and nausea are most pronounced in the morning. Symptoms shared by the entire spectrum of CAPS comprise fever, chills, conjunctivitis, diffuse urticaria, headaches and myalgia/arthralgia. The intensity of headaches may reflect the intensity of CNS disease, especially the chronic meningitis encountered in patients with CAPS. The course of the disease and the disease-associated disability varies among patients with CAPS. Those with FCAS primarily experience episodic attacks with very little if any permanent organ damage, while patients with CINCA/NOMID usually have continuous disease resulting in debilitating organ damage. The results from the Delphi process and from the expert meeting showed consensus on daily evaluation of fever, limb pain, skin rash, conjunctivitis and headaches to assess CAPS disease activity.

Table 2 shows the selected variables for each disease. A final score was generated by calculating the sum of the scores for all variables divided by the number of days over which the diary was completed. Scores could vary from 0 to 16 (0-13 in CAPS). Serial disease activity measurement should be obtained with sufficiently long intervals (eg, 3-6 months) to detect meaningful

Table 2 Variables selected during consensus conference to assess disease activity

\begin{tabular}{llll}
\hline FMF & MKD & TRAPS & CAPS \\
\hline Fever $\geq 38^{\circ} \mathrm{C}$ & Fever $\geq 38^{\circ} \mathrm{C}$ & Fever $\geq 38^{\circ} \mathrm{C}$ & Fever $\geq 38^{\circ} \mathrm{C}$ \\
$\left(100.4^{\circ} \mathrm{F}\right)$ & $\left(100.4^{\circ} \mathrm{F}\right)$ & $\left(100.4^{\circ} \mathrm{F}\right)$ & $\left(100.4^{\circ} \mathrm{F}\right)$ \\
Abdominal pain & Abdominal pain & Abdominal pain & Limb pain \\
Arthralgia or myalgia & Nausea/vomiting & Limb pain & Conjunctivitis \\
Swelling of the joints & Diarrhoea & Eye manifestations & Headaches \\
Chest pain & Limb pain & Skin rash & Skin rash \\
Skin rash & Painful lymph nodes & Overall TRAPS & \\
& & symptoms & \\
\hline
\end{tabular}

CAPS, cryopyrin-associated periodic syndromes; FMF, familial Mediterranean fever; MKD, mevalonate kinase deficiency;TRAPS, TNF receptor-1-associated periodic syndrome. changes. All experts agreed that fatigue, quality of life and biological measures were different domains and should not be included in the disease activity score. Tables 2-6 show diaries proposed by experts for each disease. The final activity score consists of the sum of all variables $(a+b+c+d+e+f)$ divided by the number of days over which the diary was completed. Since we have developed a daily diary for the disease activity score, we decided also to add information concerning the use of rescue treatment in anticipation of a possible composite score that we will develop. The use of rescue treatment will be noted by patients in the diary but should not be used in the computation of the activity score.

\section{DISCUSSION}

The use of a disease activity score is essential in clinical trials, both to assess the efficacy of new therapies and to standardise assessments across trials. ${ }^{20} \mathrm{~A}$ widely used and validated scoring system would facilitate comparison and meta-analysis of studies in the future, which would be particularly useful in the context of these rare diseases. Unlike other inflammatory diseases, ${ }^{21}$ no activity scores for either adults or children are available for SAID, which limits the ability to assess current treatments or to appreciate whether disease activity predicts the development of serious long-term complications such as permanent hearing loss, vision loss or AA amyloidosis. In this context, a disease activity score applicable to adults and to children is of great importance. To assess the course of the disease and to detect clinically important change with respect to a therapeutic intervention, a measure must be sensitive to change. In FMF, disease severity scores were published for adults and a severity score was suggested for children, ${ }^{22-24}$ but unlike disease activity scores, severity scores include variables that do not change with time (eg, age of onset) or include damage (eg, amyloidosis).

As some clinical manifestations are similar between the SAID, the experts decided to construct activity scores which share the same format but are adapted to each disease. For all SAID, both experts and the study group considered that the duration and frequency of the attacks were important indicators of disease activity. In building these scores, the study group agreed that using a single format would facilitate standardisation and be easier to use. The scores are generated from a standardised patient diary which can easily be completed by the patient or parents. After validation, these tools could be adopted in future clinical trials, epidemiological research and/or routine clinical practice. For each SAID, the use of a prospective patient-based diary was felt to be the best way to accurately assess the major symptoms including intensity, frequency and duration. Evaluation periods for which the diary is completed can be adapted as clinically appropriate-a 3-month period survey is probably suitable for FMF

Table 3 Diary for patients with familial Mediterranean fever (FMF)

\begin{tabular}{|c|c|c|c|c|c|c|c|}
\hline \multicolumn{8}{|c|}{ FMF-related symptoms today } \\
\hline \multicolumn{3}{|l|}{ Name: } & \multicolumn{4}{|l|}{ Month: } & \multirow{2}{*}{$\begin{array}{l}\text { Year: } \\
\text { Pain relief taken }\end{array}$} \\
\hline Days & Fever $\geq 38^{\circ} \mathrm{C}\left(100.4^{\circ} \mathrm{F}\right)$ & Abdominal pain & Chest pain & Arthralgia or myalgia & Swelling of the joints & Skin rash & \\
\hline \multicolumn{8}{|l|}{1} \\
\hline \multicolumn{8}{|l|}{2} \\
\hline \multicolumn{8}{|l|}{$\begin{array}{l}\ldots \\
31\end{array}$} \\
\hline
\end{tabular}


Table 4 Diary for patients with mevalonate kinase deficiency (MKD)

\begin{tabular}{|c|c|c|c|c|c|c|c|}
\hline \multicolumn{8}{|c|}{ MKD-related symptoms today } \\
\hline \multicolumn{3}{|l|}{ Name: } & \multicolumn{4}{|l|}{ Month: } & \multirow{2}{*}{$\begin{array}{l}\text { Year: } \\
\text { Pain relief taken }\end{array}$} \\
\hline Days & Fever $\geq 38^{\circ} \mathrm{C}\left(100.4^{\circ} \mathrm{F}\right)$ & Abdominal pain & Painful lymph nodes & Limb pain & Nausea/vomiting & Diarrhoea & \\
\hline \multicolumn{8}{|l|}{1} \\
\hline \multicolumn{8}{|l|}{2} \\
\hline 31 & & & & & & & \\
\hline
\end{tabular}

Table 5 Diary for patients with tumour necrosis factor receptor-1-associated periodic syndrome (TRAPS)

\begin{tabular}{|c|c|c|c|c|c|c|c|c|}
\hline \multicolumn{7}{|c|}{ TRAPS-related symptoms today } & \multirow{2}{*}{\multicolumn{2}{|c|}{$\begin{array}{l}\text { Medication taken for TRAPS today } \\
\text { Year: }\end{array}$}} \\
\hline \multicolumn{3}{|l|}{ Name: } & \multicolumn{4}{|l|}{ Month: } & & \\
\hline Days & Fever $\geq 38^{\circ} \mathrm{C}\left(100.4^{\circ} \mathrm{F}\right)$ & Overall TRAPS symptoms & Abdominal pain & Limb pain & Skin rash & Eye manifestations & Pain relief taken & Prednisolone \\
\hline \multicolumn{9}{|l|}{1} \\
\hline \multicolumn{9}{|l|}{2} \\
\hline \multicolumn{9}{|l|}{$\cdots$} \\
\hline
\end{tabular}

Table 6 Diary for patients with cryopyrin-associated periodic syndromes (CAPS)

\begin{tabular}{|c|c|c|c|c|c|c|}
\hline \multicolumn{3}{|l|}{ Name: } & \multicolumn{3}{|l|}{ Month: } & \multirow{2}{*}{$\begin{array}{l}\text { Year: } \\
\text { Pain relief taken }\end{array}$} \\
\hline Days & Fever $\geq 38^{\circ} \mathrm{C}\left(100.4^{\circ} \mathrm{F}\right)$ or chills & Eye manifestations & Headaches & Limb pain & Skin rash & \\
\hline \multicolumn{7}{|l|}{1} \\
\hline \multicolumn{7}{|l|}{2} \\
\hline \multicolumn{7}{|l|}{$\begin{array}{l}\ldots \\
31\end{array}$} \\
\hline
\end{tabular}

and MKD but could be longer in TRAPS and shorter in CAPS. The method of calculation of the score is straightforward and provides a global score ranging from 0 to 16 (0-13 for CAPS) for the evaluation period.

Selection of the variables included in the score was based on physicians' and patients' opinion. This process had some limitations. First, the final response rate of the Delphi survey was limited as only 19 of the 65 experts approached completed both questionnaires. Among the physicians who did not answer, we hypothesised that some of them were probably not interested in the study but that most were not sufficiently confident to provide an opinion on all SAID as some of these conditions are very rare with an incidence of approximately 1 per million. Also, we did not include enough patients to be completely certain that their opinions were representative. However, the main point of this paper is the result of the consensus conference. Both combined physician (Delphi technique) and patient views (interviews) were used as a starting point for experts during the consensus conference to fuel the discussion and were not used directly for the construction of the scores.

While disease activity is important, it does not summarise the overall effect of disease on patients with SAID. During the consensus conference, experts emphasised the need to collect data on additional disease parameters, some of which could be included in a composite score. The activity score aims to be the first step in the construction of a more elaborate and complete composite score. Some standardised instruments to assess the global impact of the disease such as visual analogue scales were also proposed. The need for rescue treatment (such as corticosteroids), the results of biological measurements such as CRP and SAA, quality of life, organ-specific inflammation and disease-associated damage (including joint destruction, growth retardation, hearing and vision loss, AA amyloidosis) are important aspects in the evaluation of disease impact and the management of SAID. We aimed to include in the final composite score variables that are easily assessable and tests which are available worldwide. CRP is a widely accessible, inexpensive and reliable measure of the hepatic non-specific inflammatory acute phase response. Despite limited availability, the measurement of SAA is the current gold standard in determining the prognosis and response to treatment in studies assessing the development of AA amyloidosis. ${ }^{14}{ }^{24}$ Both blood tests, CRP and SAA can be helpful in episodic diseases if assayed between attacks since evidence of complete normalisation suggests control of subclinical inflammation. ${ }^{25}$ Evaluation of fatigue, a very common symptom emphasised by patients and experts, as well as the assessment of quality of life are major parameters that could be included in a composite score. Existing quality of life 
measures such as those used in rheumatological practice may have to be adapted and validated or a new specific scale created. In the same way, global evaluation of disease activity by patients and the use of rescue treatments are of major importance in the management of SAID. In addition, disease-specific organ inflammation and disease-associated damage predicts long-term health-related and more general patient outcomes and are of importance in assessing disease severity and longterm efficacy. Our consensus effort focused on the clinical activity score.

In conclusion, using standardised consensus formation techniques, we have developed a preliminary scoring system to measure disease activity in four hereditary autoinflammatory disorders. We propose a single-format disease-adapted patient diary-based disease activity score which could be used in clinical research and possibly in clinical practice. In a next step we propose to conduct a prospective validation phase. For this, we will solicit centres participating in this study as well as other centres located in countries in which SAID are common.

Acknowledgements The authors thank Professor Isabelle Touitou, Dr Sandrine Lacassagne and Mr Khaoussou Sylla.

Funding sources This study was supported by grants from the French Ministry of Health (PHRC 2008) and Assistance Publique-Hôpitaux de Paris (APHP).

\section{Competing interests None.}

Provenance and peer review Not commissioned; externally peer reviewed.

Author affiliations ${ }^{1}$ Department of Pediatrics and Pediatric Rheumatology, National Reference Center for Auto-Inflammatory Disorders, Bicêtre University Hospital, Paris XI University, Le Kremlin-Bicêtre, France

${ }^{2}$ Department of Pediatrics, University Medical Center Utrecht, Utrtecht, The Netherlands

${ }^{3}$ UO Pediatria II and Department of Pediatrics, "G Gaslini" Institute and University of Genoa, Italy

${ }^{4}$ Department of Pediatrics, Hacettepe University Faculty of Medicine, Ankara, Turkey ${ }^{5}$ National Amyloidosis Centre, University College London Medical School, London, UK ${ }^{6}$ National Institute of Arthritis Musculoskeletal and Skin Disease, NIH, Bethesda, Maryland, USA

${ }^{7}$ Department of Pediatrics, National Reference Center for Auto-Inflammatory Disorders, A Mignot Hospital, Versailles, France

${ }^{8}$ Department of Pediatric Immuno-Hematology and Pediatric Rheumatology, NeckerEnfants Malades Hospital, Paris, France

${ }^{9}$ Department of Internal Medicine, National Reference Center for Inflammatory Amyloidosis and Familial Mediterranean Fever, Tenon University Hospital, Université Pierre et Marie Curie Paris 6, Paris, France

${ }^{10}$ Department of General Internal Medicine, Radboud University Nijmegen Medical Centre, Nijmegen, The Netherlands

${ }^{11}$ Division of Pediatric Rheumatology, Department of Pediatrics, University Hospital Tübingen, Germany

${ }^{12}$ Department of Pediatrics and Medicine, University of California, San Diego, Rady Children's Hospital of San Diego, San Diego, California, USA

${ }^{13}$ Department of Infectious Diseases and Immunology, University Children's Hospital, Munich, Germany

14Department of Nephrology and Pediatric Rheumatology, Hôpital Femme Mère Enfant, Hospices Civils de Lyon, Lyon, France

${ }^{15}$ Department of Pediatrics, Children's Hospital of Bordeaux, France

${ }^{16}$ Department of Internal Medicine, European Georges Pompidou Hospital, Paris-

Descartes University, Paris, France

\section{REFERENCES}

1. Touitou I, Koné-Paut I. Autoinflammatory diseases. Best Pract Res Clin Rheumatol 2008;22:811-29

2. Lachmann HJ, Kone-Paut I, Kuemmerle-Deschner JB, et al. Use of canakinumab in the cryopyrin-associated periodic syndrome. N Engl J Med 2009;360:2416-25.

3. Hoffman HM, Throne ML, Amar NJ, et al. Efficacy and safety of rilonacept (interleukin-1 Trap) in patients with cryopyrin-associated periodic syndromes: results from two sequential placebo-controlled studies. Arthritis Rheum 2008;58:2443-52.

4. Sinha I, Jones L, Smyth RL, et al. A systematic review of studies that aim to determine which outcomes to measure in clinical trials in children. PLoS Med 2008;5:e96.

5. Drenth JP, van der Meer JW. Hereditary periodic fever. N Eng/ J Med 2001;345:1748-57

6. Batthish M, Schneider R, Ramanan AV, et al. What does "active disease" mean? patient and part5ent perceptions of disease activity in the systemic arthritis form of juvenile idiopathic arthritis (SO-JIA). Rheumatology (Oxford) 2005;44:796-9.

7. Ruperto N, Ravelli A, Murray KJ, et al. Preliminary core sets of measures for disease activity and damage assessment in juvenile systemic lupus erythematosus and juvenile dermatomyositis. Rheumatology (Oxford) 2003:42:1452-9.

8. Ruperto N, Meiorin S, lusan SM, et al. Consensus procedures and their role in pediatric rheumatology. Curr Rheumatol Rep 2008;10:142-6.

9. Jones J, Hunter D. Consensus methods for medical and health services research. BMJ 1995;311:376-80.

10. Zulian F, Woo P, Athreya BH, et al. The Pediatric Rheumatology European Society/ American College of Rheumatology/European League against Rheumatism provisional classification criteria for juvenile systemic sclerosis. Arthritis Rheum 2007:57:203-12.

11. Kallinich T, Haffner D, Niehues T, et al. Colchicine use in children and adolescents with familial Mediterranean fever: literature review and consensus statement. Pediatrics 2007;119:e474-83.

12. Livneh A, Langevitz P, Zemer D, et al. The changing face of familial Mediterranean fever. Semin Arthritis Rheum 1996;26:612-27.

13. Livneh A, Langevitz P, Zemer D, et al. Criteria for the diagnosis of familial Mediterranean fever. Arthritis Rheum 1997:40:1879-85

14. Samuels J, Ozen S. Familial Mediterranean fever and the other autoinflammatory syndromes: evaluation of the patient with recurrent fever. Curr Opin Rheumatol 2006;18:108-17.

15. Tunca M, Akar S, Onen F, et al. Familial Mediterranean fever (FMF) in Turkey: results of a nationwide multicenter study. Medicine (Baltimore) 2005;84:1-11.

16. Yalçinkaya F, Ozen S, Ozçakar ZB, et al. A new set of criteria for the diagnosis of familial Mediterranean fever in childhood. Rheumatology (Oxford) 2009;48:395-8.

17. van der Hilst JC, Bodar EJ, Barron KS, et al. Long-term follow-up, clinical features, and quality of life in a series of 103 patients with hyperimmunoglobulinemia D syndrome. Medicine (Baltimore) 2008;87:301-10.

18. Dodé $\mathbf{C}$, André $\mathrm{M}$, Bienvenu T, et al. The enlarging clinical, genetic, and population spectrum of tumor necrosis factor receptor-associated periodic syndrome. Arthritis Rheum 2002;46:2181-8.

19. Aksentijevich I, D Putnam C, Remmers EF, et al. The clinical continuum of cryopyrinopathies: novel CIAS1 mutations in North American patients and a new cryopyrin model. Arthritis Rheum 2007;56:1273-85.

20. Singh JA, Solomon DH, Dougados $M$, et al. Development of classification and response criteria for rheumatic diseases. Arthritis Rheum 2006;55:348-52.

21. Consolaro A, Ruperto N, Bazso A, et al. Development and validation of a composite disease activity score for juvenile idiopathic arthritis. Arthritis Rheum 2009;61:658-66.

22. Mor A, Shinar Y, Zaks N, et al. Evaluation of disease severity in familial Mediterranean fever. Semin Arthritis Rheum 2005;35:57-64.

23. Pras $\mathbf{E}$, Livneh A, Balow JE, Jr, et al. Clinical differences between North African and Iraqi Jews with familial Mediterranean fever. Am J Med Genet 1998;75:216-9.

24. Ozen S, Aktay N, Lainka $E$, et al. Disease severity in children and adolescents with familial Mediterranean fever: a comparative study to explore environmental effects on a monogenic disease. Ann Rheum Dis 2009;68:246-8.

25. Gillmore JD, Lovat LB, Persey MR, et al. Amyloid load and clinical outcome in AA amyloidosis in relation to circulating concentration of serum amyloid A protein. Lancet 2001;358:24-9. 